\title{
Low noise amplifier R\&D for CULTASK experiment at CAPP
}

\section{Doyu Lee*}

Center for Axion and Precision Physics Research, Institute for Basic Science, Daejeon, Korea Department of Physics, Korea Advanced Institute of Science and Technology, Daejeon, Korea E-mail: du5698@kaist.ac.kr

Woohyun Chung ${ }^{1}$, Ohjoon Kwon ${ }^{1}$, Andrei Matlashov ${ }^{1}$, Jinsu Kim ${ }^{1,2}$, Danho Ahn ${ }^{1,2}$, Caglar Kutlu $^{1,2}$, JihnE Kim ${ }^{1,3}$, Yannis K. Semertzidis ${ }^{1,2}$

${ }^{1}$ Center for Axion and Precision Physics Research, Institute for Basic Science, Daejeon, Korea

${ }^{2}$ Department of Physics, Korea Advanced Institute of Science and Technology, Daejeon, Korea

${ }^{3}$ Department of Physics, Kyung Hee University, Seoul 02447, Korea

IBS/CAPP has launched CAPP-PACE, direct axion detection experiment aimed at a frequency range of $2.45-2.75 \mathrm{GHz}$. In the present detector setup, we utilize $1 \mathrm{~K}$ HEMT amplifiers which are the world-best commercial available silicon-based low temperature amplifier. However, in order to reach the QCD axion sensitivity and speed up the experiment, we are currently examining the possibility of replacing the first stage HEMT with a SQUID amplifier which could reduce the noise temperature drastically. A MSA (Microstrip SQUID Amplifier) is a good option for achieving a low noise level. This poster includes information of MSA, gain and noise measurement, and test results under magnetic fields. The finalized RF receiver chain of our experiment with a MSA will be also presented.

The 39th International Conference on High Energy Physics (ICHEP2018)

4-11 July, 2018

Seoul, Korea

${ }^{*}$ Speaker. 
The CAPP-PACE experiment is the first microwave cavity-based axion search experiment in Korea which was designed according to the scheme proposed by Sikivie in 1983[1]. We search for axions with target frequencies between $2.45 \mathrm{GHz}$ to $2.75 \mathrm{GHz}$ using a $12 \mathrm{~cm}$ bore $8 \mathrm{~T}$ superconducting magnet and a dilution refrigerator. We started to run our first experiment from early 2018 and successfully took the data. Now we are upgrading our detector. A $25 \mathrm{~T}$ magnet from BNL will be introduced in early 2020. A superconducting cavity R\&D is underway to achieve higher quality factor. Among those efforts, a low noise amplifier R\&D is especially important. As can be inferred from the fact that a axion is a strong candidate for the dark matter, its coupling strength to the photon is extremely weak. Therefore a low noise level of preamplifier is a essential part for the fast scan of the wide axion frequency ranges.



Figure 1: Scan rate according to the system noise temperature of RF chain.

We got our first data using the best commercially available HEMT amplifiers which have the noise temperature of $1 \mathrm{~K}$. At the same time, we made a bypass line for R\&D of quantumlimited-noise amplifier with a MSA(Microstrip SQUID Amplifier) to reach the SQL(Standard Quantum Limit) level. A MSA is an amplifier using the flux-to-voltage transfer characteristics

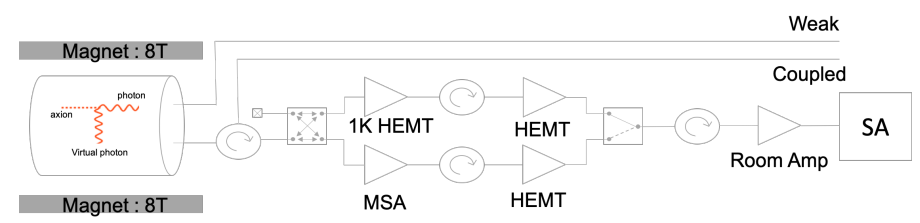

Figure 2: Simplified read-out chain of the CAPP-PACE experiment.

of SQUID(Superconducting Quantum Interference Device)[2]. It can operate at GHz frequencies with the micro-strip resonator architecture and theoretically its noise can reach close to the SQL. We are testing MSAs coming from various groups such as KRISS(Korea), IPHT(Germany), ezSQUID(Germany). With those quantum-limited amplifiers we can reach the next level soon.

\section{Acknowledgement}

This work was supported by IBS-R017-D1-2018-a00 in the Republic of Korea.

\section{References}

[1] P. Sikivie, Phys. Rev. Lett. 51, 1415 (1983) Erratum: [Phys. Rev. Lett. 52, 695 (1984)]. doi:10.1103/PhysRevLett.51.1415, 10.1103/PhysRevLett.52.695.2

[2] Andrei Matlashov, Matthias Schmelz, Vyacheslav Zakosarenko, Ronny Stolz, and Yannis K. Semertzidis. Squid amplifiers for axion search experiments. Cryogenics, 91:125 - 127, 2018. 